\title{
Collapse and Fragmentation of Magnetic Molecular Cloud Cores with the Enzo AMR MHD Code. I. Uniform Density Spheres
}

\author{
Alan P. Boss and Sandra A. Keiser \\ Department of Terrestrial Magnetism, Carnegie Institution for Science, 5241 Broad Branch \\ Road, NW, Washington, DC 20015-1305 \\ boss@dtm.ciw.edu
}

\begin{abstract}
Magnetic fields are important contributers to the dynamics of collapsing molecular cloud cores, and can have a major effect on whether collapse results in a single protostar or fragmentation into a binary or multiple protostar system. New models are presented of the collapse of magnetic cloud cores using the adaptive mesh refinement (AMR) code Enzo2.0. The code was used to calculate the ideal magnetohydrodynamics (MHD) of initially spherical, uniform density and rotation clouds with density perturbations, i.e., the Boss \& Bodenheimer (1979) standard isothermal test case for three dimensional (3D) hydrodynamics (HD) codes. After first verifying that Enzo reproduces the binary fragmentation expected for the non-magnetic test case, a large set of models was computed with varied initial magnetic field strengths and directions with respect to the cloud core axis of rotation (parallel or perpendicular), density perturbation amplitudes, and equations of state. Three significantly different outcomes resulted: (1) contraction without sustained collapse, forming a denser cloud core, (2) collapse to form a single protostar with significant spiral arms, and (3) collapse and fragmentation into binary or multiple protostar systems, with multiple spiral arms. Comparisons are also made with previous MHD calculations of similar clouds with barotropic equations of state. These results for the collapse of initially uniform density spheres illustrate the central importance of both magnetic field direction and field strength for determining the outcome of dynamic protostellar collapse.
\end{abstract}

Subject headings: hydrodynamics - ISM: clouds - ISM: kinematics and dynamics - MHD — stars: formation 


\section{Introduction}

Observations of dark cloud cores have shown that magnetic fields are often an important contributer to cloud support against collapse for densities in the range of $10^{3}-10^{4} \mathrm{~cm}^{-3}$ (e.g., Troland \& Crutcher 2008; Crutcher 2012). As a result, it has become increasingly routine for theoretical models of protostellar system formation to include magnetic fields. Boss (1997) used a pseudo-MHD 3D gravitational hydrodynamics code with radiative transfer in the Eddington approximation to study the collapse and fragmentation of magnetic clouds, including the effects of ambipolar diffusion. More recently, true MHD codes have become the standard. E.g., Banerjee \& Pudritz (2006) considered the collapse of a magnetized cloud core, finding that the cloud was still able to collapse and fragment into a close binary protostar system, while Price \& Bate (2007) found that magnetic pressure was more important for inhibiting binary fragmentation than either magnetic tension or braking. Machida et al. (2008) found that binary fragmentation could occur provided that the initial magnetic cloud core rotated fast enough, while Hennebelle \& Fromang (2008) and Hennebelle \& Teyssier (2008) found that magnetic clouds could fragment if an initial density perturbation was large enough. Joos et al. (2012) found that the initial direction of the magnetic field with respect to the rotation axis had an important effect on the outcome of the collapse.

Hennebelle et al. (2011) pointed out that while magnetic fields are important for cloud collapse, numerical convergence of MHD codes is difficult to achieve, illustrating the importance of careful testing (and eventually cross-comparison) of MHD code results. This paper is directed toward performing a variety of new code tests and new code comparisons with the Enzo AMR MHD code, in order to gain the necessary confidence to proceed with more realistic models of the collapse of dense molecular cloud cores (e.g., Machida et al. 2008; Duffin \& Pudritz 2009), and in particular to learn if a previous series of pseudo-MHD models of magnetic field effects on cloud collapse (Boss 2009, 2007, 2005,.., 1997) is valid. Price \& Bate (2007) and Bürzle et al. (2011) considered the collapse of spherical, magnetic cloud cores with initially uniform density and uniform magnetic field strengths, the MHD version

of the standard isothermal test case of Boss \& Bodenheimer (1979). Here we perform a number of similar MHD calculations, with the goal of comparing the Enzo AMR MHD results with these previous results. However, we begin by first testing the Enzo code on the Boss \& Bodenheimer (1979) test case, and comparing the Enzo results with those obtained by a variety of previous studies (e.g., Burkert \& Bodenheimer 1993; Myhill \& Boss 1993; Bate et al. 1995; Sigalotti 1997; Truelove et al. 1998; Kitsionis \& Whitworth 2002; Arreaga-Garcia et al. 2007). 


\section{Numerical Methods}

Protostellar collapse calculations by necessity must cover variations of many orders of magnitude in cloud density and length scales, from pc-sized molecular cloud cores to AUsized first protostellar cores. Early 3D hydrodynamic codes (e.g., Boss \& Bodenheimer 1979) used numerical grids that contracted in the radial direction as the cloud collapsed in order to maintain adequate spatial resolution. More recently, AMR codes have been developed, which allow numerical grid points to be inserted wherever they might be needed, and to be removed wherever they are not needed, for the most efficient calculation of problems with strongly varying length scales. A number of such AMR codes exist at present and many are available for public downloading (e.g., FLASH - Fryxell et al. 2000; RIEMANN - Balsara 2001; RAMSES - Teyssier 2002; NIRVANA - Ziegler 2008, AstroBEAR - Cunningham et al. 2009; Enzo - Collins et al. 2010; CHARM - Miniati \& Martin 2011; CRASH - van der Holst et al. 2011; AZEuS - Ramsey et al. 2012; PLUTO - Mignone et al. 2012).

The Enzo2.0 code is used here, for the following reasons. Enzo performs hydrodynamics (HD) using any one of three different algorithms: (1) the piecewise parabolic method (PPM) of Colella \& Woodward (1984), (2) the ZEUS method of Stone \& Norman (1992), or (3) a Runge-Kutta third-order-based MUSCL ("monotone upstream-centered schemes for conservation laws") algorithm based on the Godunov (1959) shock-handling HD method. Enzo is designed for handling strong shock fronts, which occur late in the protostellar collapse phase. This involves solving the Riemann problem (e.g., Godunov 1959) for discontinuous solutions of a fluid quantity that should be conserved. Enzo's default Riemann solver is that of Harten-Lax-van Leer (HLL), though other solvers are available as well. We used MUSCL HD with the HLL Riemann solver for all of the MHD models.

Enzo 2.0 also performs ideal magnetohydrodynamics (MHD), using the Dedner et al. (2002) divergence cleaning method (Wang et al. 2008) in concert with the MUSCL HD method noted above. Wang \& Abel (2009) have shown that while the Dedner MHD method is unable to exactly conserve the magnetic flux (i.e., keep the divergence of the field equal to zero), in practice the non-zero field divergence that arises during a typical calculation is not large enough to be dynamically important. Other versions of Enzo employ the constrained transport MHD method for preserving the zero divergence of the magnetic field (Collins et al. 2010).

Enzo2.0 has been extensively tested on a wide variety of MHD test problems and found to perform well. Enzo solves for the gravitational fields within the cloud by using a Fast Fourier Transform method for the root grid and multigrid relaxation on subgrids. Enzo also includes the option of employing flux-limited diffusion approximation radiative transfer, needed for future comparisons with previous pseudo-MHD models (e.g., Boss 2009). 
Enzo2.0 includes about 100 possible test cases, one of which is the Boss \& Bodenheimer (1979) 3D calculation for molecular cloud collapse and fragmentation, a convenient starting point for testing Enzo's ability to handle collapse calculations. The results of this test case with the Enzo2.0 code have not been published to date. In fact, this test case will not run successfully when initialized using the parameters provided in the Enzo2.0 code: either the hydrodynamic method to be used needs to be changed from the method involving the Dedner et al. (2002) MHD algorithm, or else the "dual energy formalism" switch must be disabled. In this paper we thus provide the first evaluation of Enzo2.0 on this test case, and we also extend this basic collapse test case to consider the smaller initial amplitude density perturbation considered by Burkert \& Bodenheimer (1999), as well as higher spatial resolution. Given that Enzo is a Cartesian coordinate code, it can conserve linear momentum, but not necessarily angular momentum, unlike codes written in spherical or cylindrical coordinates (e.g., Boss \& Bodenheimer 1979). Hence we also test here Enzo's ability to conserve angular momentum.

Most of the Enzo models presented here were initialized on a 3D Cartesian grid with 32 grid points in each direction, while one model used 64 grid points in each direction. A maximum of 6 levels of refinement was permitted as needed during the collapses, with a factor of 2 refinement occurring for each level, so that the maximum possible effective grid resolution was $2^{6}=64$ times higher than the initial resolution of $32^{3}$ or $64^{3}$, i.e., $2048^{3}$ or $4096^{3}$, respectively. Refinement was performed whenever necessary to ensure that the Jeans length constraint (e.g., Truelove et al. 1997) was satisfied by a factor of 8; in one test case, this was increased to being satisfied by a factor of 16 . After the models were run, a bug was revealed in the Enzo2.0 code regarding the gas mean molecular weight, which effectively reduced the gas temperature from $10 \mathrm{~K}$ to $3 \mathrm{~K}$. [Greg Bryan informed us that this error in the parameter reading code of Enzo was corrected in the main Enzo repository by Elizabeth Tasker in June 2011, shortly after we downloaded our copy of the Enzo files.] Because the temperature is not a primary variable in Enzo, this bug had no direct effect on the hydrodynamics, except that the Jeans length constraint that was actually enforced, which is calculated using the temperature, was then a factor of $\sim 2$ times more conservative than if the temperature had been correct. The effective Jeans length constraints were then satisfied by factors of either $\sim 16$ or $\sim 32$ as a result. By chance, this accidentally improved satisfaction of the Jeans length constraint compares favorably with the results of a recent study of AMR MHD (Federrath et al. 2011), which suggested that the Jeans length be resolved by at least 30 cells in order to adequately resolve turbulence excited by gravitational collapse.

Periodic boundary conditions were applied on each face of the grid's cubic box. The maximum number of Green's functions used to calculate the gravitational potential was 10 . The time step used was 0.3 of the limiting Courant time step. The results were analyzed with the yt astrophysical analysis and visualization toolkit (Turk et al. 2011). 


\section{Initial Conditions}

The various models studied here all begin with variations on the initial conditions first studied by Boss \& Bodenheimer (1979) in what has come to be known as the "standard isothermal test case" for 3D hydrodynamics codes. The initial cloud is a uniform density $\left(\rho=1.44 \times 10^{-17} \mathrm{~g} \mathrm{~cm}^{-3}\right)$, uniform temperature $(T=10 \mathrm{~K})$ sphere with radius $R=3.2 \times 10^{16}$ $\mathrm{cm}$, in solid body rotation around the $\hat{z}$ axis at an angular velocity $\Omega=1.6 \times 10^{-12} \mathrm{rad} \mathrm{s}^{-1}$. The cloud is embedded within an active computational AMR grid with a linear dimension of $3.2 \times 10^{17} \mathrm{~cm}$, i.e., a 3D box with sides 10 times larger than the cloud radius. The initial cloud has a mass $M=1.0 M_{\odot}$, composed of molecular hydrogen (mean molecular weight $\mu=2.0$ ), an initial ratio of thermal to gravitational energy of $\alpha_{i}=0.25$, and an initial ratio of rotational to gravitational energy of $\beta_{i}=0.20$.

While a uniform density sphere is clearly a highly artificial representation of a molecular cloud core, the Boss \& Bodenheimer (1979) initial conditions are nevertheless easy to reproduce for inter-code test comparisons, which was the main motivation for their paper. These initial conditions were also motivated by the earlier Black \& Bodenheimer (1976) axisymmetric, two dimensional (2D) rotating cloud collapse calculations, which found that rings might form, which were themselves likely to fragment into multiple protostar systems in a fully 3D calculation. Boss \& Bodenheimer (1979) found that when given an initial 50\% azimuthal $(\cos (m \phi)$, with $m=2)$ density perturbation, the perturbation grew and fragmented the cloud into a binary protostar system. Isothermal thermodynamics with a temperature of $T=10 \mathrm{~K}$ was assumed throughout the collapse.

The Boss \& Bodenheimer (1979) standard isothermal test case, or a slight variation with an initial $10 \%$ azimuthal density perturbation, has been often used to test 3D hydro codes (e.g., Burkert \& Bodenheimer 1993; Myhill \& Boss 1993; Bate et al. 1995; Sigalotti 1997; Truelove et al. 1998; Kitsionis \& Whitworth 2002; Arreaga-Garcia et al. 2007). A nonisothermal test case for protostellar collapse also exists, calculated with two different 3D radiative hydrodynamics codes by Myhill \& Boss (1993), where compressional heating and radiative cooling (in either the Eddington or the diffusion approximation) are taken into account. In order to gain insight into how MHD fragmentation might be affected by nonisothermal thermodynamics, a set of MHD models has been calculated where the isothermal approximation is replaced by the "barotropic" equation employed by, e.g., Price \& Bate (2007) and Bürzle et al. (2011) in their 3D MHD models of the collapse of uniform density spheres. The barotropic pressure $p$ is assumed to depend on the gas density $\rho$ as $p=K \rho^{\gamma}$, where the polytropic exponent $\gamma$ equals 1 for $\rho<10^{-14} \mathrm{~g} \mathrm{~cm}^{-3}$ and $7 / 5$ for

$\rho>10^{-14} \mathrm{~g} \mathrm{~cm}^{-3}$, mimicking the effects of nonisothermal thermodynamics. $K=c_{s}^{2}$, where $c_{s}$ is the isothermal sound speed $\left(0.2 \mathrm{~km} \mathrm{~s}^{-1}\right)$, below the critical density of $\rho_{c}=10^{-14}$ 
$\mathrm{g} \mathrm{cm}^{-3}$, while for $\rho>\rho_{c}, K=c_{s}^{2} \rho_{c}^{-2 / 5}$, ensuring continuity of the pressure across the critical density. While a convenient approximation for code inter-comparisons, the barotropic approximation does not necessarily lead to the same outcome as a collapse calculated with a full thermodynamical treatment, including Eddington approximation radiative transfer (e.g., Boss et al. 2000), so some caution is warranted when drawing conclusions from such models.

The models with magnetic fields all began with the same initial conditions as in the Boss \& Bodenheimer test case, with initial density perturbations of either $10 \%$ or $50 \%$, and with initially straight magnetic fields that were aligned with either the $\hat{x}$ or $\hat{z}$ axis (i.e., either perpendicular or parallel to the cloud's rotation axis, respectively). Table 2 lists the initial conditions and basic results for the MHD models with the isothermal equation of state, while Tables 3 and 4 refer to the MHD models with the barotropic equation of state and initial $50 \%$ or $10 \%$ density perturbations, respectively. The results columns describe the midplane $(z=0)$ density configurations at the final time computed $t_{f}$, expressed in terms of the initial uniform density cloud free fall time $t_{f f}=(3 \pi / 32 G \rho)^{1 / 2}=1.76 \times 10^{4} \mathrm{yr}$, where $G$ is the gravitational constant and $\rho=1.44 \times 10^{-17} \mathrm{~g} \mathrm{~cm}^{-3}$.

\section{Isothermal HD Results}

Three non-magnetic, isothermal models were computed, which were either identical to Boss \& Bodenheimer (1979) [model BB79], or were a variation on the standard isothermal test case: model BB79-10, where the initial density perturbation was $10 \%$ instead of $50 \%$, and model BB79-10-HR, where the initial grid was $64^{3}$ instead of $32^{3}$ and the Jeans length constraint was improved by another factor of 2 .

Figure 1 shows the evolution of model BB79. The large initial density perturbation seen in Figure 1a collapses and fragments directly into the binary protostar system seen in Figure $1 \mathrm{~d}$, at a maximum density (nearly $\sim 10^{-11} \mathrm{~g} \mathrm{~cm}^{-3}$ ) that is considerably higher than the maximum density for which the isothermal approximation is valid, i.e., beyond $\rho_{c}=10^{-14} \mathrm{~g}$ $\mathrm{cm}^{-3}$. This evolution is quite similar to that previously obtained using a variety of 3D HD codes (e.g., Myhill \& Boss 1993; Burkert \& Bodenheimer 1993; Bate, Bonnell \& Price 1995; Sigalotti 1997).

A different evolution results when the initial density perturbation is reduced from $50 \%$ (model BB79) to 10\% (model BB79-10). As first found by Burkert \& Bodenheimer (1993), the initial density perturbation forms only a transient binary that merges into a thin bar capable of fragmenting into multiple objects. Truelove et al. (1998) found that the thin bar continued to collapse to very high densities $\left(\sim 10^{-10} \mathrm{~g} \mathrm{~cm}^{-3}\right)$ and formed two singular 
filaments. Similar thin bars were found by Kitsionis \& Whitworth (2002) and by ArreagaGarcia et al. (2007) with their smoothed particle hydrodynamics (SPH) codes.

Figure 2 shows the evolution of model BB79-10. As a result of the smaller initial density perturbation, the collapse and fragmentation process proceeds slightly slower than in model BB79, and the binary that forms (Figure 2b) is weakly defined compared to model BB79. The binary fragments begin to merge together (Figure 2c) into a bar-like structure, which then collapses further to form a thin, intermediate filament (Figure 2d), similar to those previously found with other high-resolution HD codes.

Finally, we consider model BB79-10-HR, with the spatial resolution increased by a factor of two in all three directions compared to model BB79-10, along with an improved Jeans length constraint. This model collapses in much the same manner as model BB79-10, implying that the collapse is reasonably well-resolved to the phase of formation of the thin filament. Figure 3 shows that at this phase of evolution, both models are quite similar, though the structure in model BB79-10-HR is clearly better-defined than in model BB79-10, as expected.

Enzo is designed to conserve mass and linear momentum, but not angular momentum. The degree to which mass and angular momentum are conserved depends on the hydrodynamic method (HM) used, as well as on other parameters in the Enzo input file. We have tested all three of the possible Enzo hydrodynamics algorithms (see Numerical Methods section) for mass and angular momentum conservation on the Boss \& Bodenheimer (1979) test case. The results are listed in Table 1. Model BB79 was run using the ZEUS method (Stone \& Norman 1992), model BB79-HM-0 with the PPM method (Colella \& Woodward 1984), and model BB79-HM-4 with the Runge-Kutta MUSCL Godunov (1959) method, denoted simply by MUSCL. Models BB79-10 and BB79-10-HR were also run with the MUSCL method. Table 1 shows that the total mass $M_{\text {tot }}$ and total angular momentum $J_{\text {tot }}$ are reasonably well conserved with all three methods, though the exact reason for even these relatively modest losses is unclear, given that other 3D hydro codes are able to conserve mass and angular momentum to machine precision (e.g., Boss 1997). The most likely cause is the interpolation that is used to create new sub-grids and remove old sub-grids. The models in Table 1 all used Enzo's "second-order accurate" interpolation methods, which seemed to perform the best on this problem. When Enzo's "first-order accurate" interpolation was used, $4 \%$ of the mass and $14 \%$ of the angular momentum was lost for BB79, compared to $0.7 \%$ and $5 \%$, respectively, for the "second-order accurate" interpolation method. With the use of this latter interpolation method, Table 1 shows that the MUSCL hydrodynamics method is acceptable, and these methods were used for all of the MHD models in the remainder of this paper.

We conclude that Enzo is able to reliably reproduce previously agreed-upon 3D HD 
results for the standard isothermal test case and its variations, and that the initial spatial resolution used $\left(32^{3}\right)$ for these exploratory models is sufficient to give an adequate indication of the likelihood of fragmentation during the isothermal collapse regime.

\section{Isothermal MHD Results}

We now turn to the effects of frozen-in magnetic fields on the collapse and fragmentation of initially uniform density, isothermal clouds. Tables 2 and 3 summarize the initial conditions and outcomes of these models, which started from initial conditions identical to either model BB79 (Table 2) or to model BB79-10 (Table 3), but with an initially straight magnetic field that was either parallel $\left(B_{z} \neq 0\right)$ or perpendicular $\left(B_{x} \neq 0\right)$ to the initial rotation axis $(\hat{z})$. Given that model BB79 clearly fragmented into at least a binary protostar system (Figure 1), while model BB79-10 formed a thin bar, these two sets of models provide a convenient means to assess the overall effects of frozen-in magnetic fields on the collapse and fragmentation of dense cloud cores.

Tables 2 and 3 also list the initial mass to flux ratios $(M / \Phi)$ for the models, given in units of the nominal critical ratio for stability, based on the values given by Price \& Bate (2007). Thus models with $M / \Phi>1$ are magnetically supercritical and nominally unstable to gravitational collapse, while models with $M / \Phi<1$ are magnetically subcritical and nominally stable to gravitational collapse.

\subsection{BB79 MHD Results}

Three distinct outcomes characterize the magnetic cloud models: (1) contraction over a few orders of magnitude increase in density, without sustained collapse, resulting in an un-fragmented, though considerably denser, magnetic cloud core, (2) sustained collapse to form a single protostar with significant spiral arms, and (3) collapse and fragmentation into binary or multiple protostar systems, with multiple spiral arms. In the language of magnetic field theory (e.g., Crutcher 2012), evidently outcome (1) involves a magnetically subcritical cloud, while outcomes (2) and (3) involve magnetically supercritical clouds.

Figure 4 shows the evolution of model mag-z-300, which evolved the closest to model BB79, given that this model had the lowest initial magnetic field strength of the $B_{z} \neq 0$ models, coupled with the fact that the $B_{z} \neq 0$ models allow for the gas to collapse unimpeded along the magnetic field lines onto the $z=0$ midplane, which is also favored by rotation about the $\hat{z}$ axis. Compared to Figure 1, Figure 4 shows that the magnetic field prevents the 
initial density perturbations from growing as rapidly as in BB79, allowing the perturbations to fall inward closer together (Figure 4b) before forming two thin filaments (Figure 4c) which ultimately fragment into a binary or multiple protostellar system (Figure 4d), accompanied by a large number of spiral arms and moderate density clumps. While the magnetic field is able to slow the growth of the density perturbation, forcing it into an intermediate thin filament similar to that produced in BB79-10, in the end the frozen-in magnetic field is not strong enough to prevent the cloud from fragmenting. By the time this fragmentation occurs, however, the maximum density $\left(>10^{-12} \mathrm{~g} \mathrm{~cm}^{-3}\right)$ exceeds that where the isothermal approximation remains valid, so this result should not be taken as fully realistic.

Model mag-z-300 can be used to test the degree to which Enzo conserves magnetic flux, as should be the case for ideal hydrodynamics. This is accomplished by monitoring the divergence of the magnetic field ("DivB"), which starts equal to zero everywhere in the cloud, and should remain so throughout the evolution. Requiring a zero divergence of magnetic field is equivalent to stating that there are no magnetic charges, in contrast to electric charges. In practice, the Dedner el al. (200-2) MHD method does not keep DivB $=0$, but Enzo k.eps DivB small enough that the errors that are introduced as a result are not severe (Wang \& Abel 2009). We have tested this assertion for model mag-z-300 by monitoring DivB throughout the evolution depicted in Figure 4. For the initial cloud (Figure $4 \mathrm{a}$ ), DivB $=0$ everywhere, as expected. As the evolution proceeds, DivB begins to grow, first at the edge of the cloud, and then later at the boundaries of new sub-grids as the collapse proceeds (Figure 4b). By the time that fragmentation has occurred (Figure $4 \mathrm{c}, \mathrm{d})$, DivB peaks at the locations of the density and magnetic field maxima. The dynamical importance of non-zero DivB can be assessed by calculating the ratio of DivB to the ratio of the magnetic field strength divided by the local grid spacing, as was done by Wang \& Abel (2009), i.e., $\nabla \cdot B \Delta x / B$. This critical ratio should be less than one if the MHD solution is to be reasonably accurate (Wang \& Abel 2009). When evaluated for the highest peaks of DivB for model mag-z-300 (Figure 4 d), $\nabla \cdot B \Delta x / B \sim<0.1$, showing that, while not perfect, Enzo2.0 is able to conserve the magnetic flux reasonably well.

Figure 5 shows the results for two models that illustrate the other two possible outcomes noted above: model mag-z-1600, which collapses to form a single protostar with spiral arms, and model mag-z-1800, which contracts but does not collapse. These two models demonstrate the dramatic difference in outcome produced by a relatively small change in the initial magnetic field strength: evidently these two models span a critical value for dynamic collapse versus minor contraction, for the assumed initial conditions.

Figure 6 shows the evolution of model mag-x-300, identical to model mag-z-300 except for the initial orientation of the magnetic field. While both models fragment, the evolutions 
are significantly different, as can be seen by comparing Figures 4 and 6 . Because gas can flow along the field lines aligned initially with the $\hat{x}$ axis in model mag-x-300, Figure $6 \mathrm{~b}$ shows that the initial density perturbations (which are also aligned initially with the $\hat{x}$ axis) grow preferentially along the $\hat{x}$ axis direction, compared to Figure $4 \mathrm{~b}$ for model mag-z-300, where the growth is more in the radial direction. This leads the formation of two off-set, nearly parallel filaments in the former model (Figure 6c), compared to two filaments with a linear alignment in the latter (Figure 4c). The subsequent fragmentation of the filaments in either case leads to a binary or multiple protostellar system (Figures $4 \mathrm{~d}$ and 6d), though with distinct overall differences at this early phase of evolution.

Model mag-x-300 has also been tested for magnetic flux conservation, as was done for mag-z-300, and the ratio $\nabla \cdot B \Delta x / B$ was found to be no worse than 0.1 , again implying a reasonably accurate MHD solution.

Figure 7 compares the results for models mag-x-800 and mag-x-1000: the former collapses to form a single protostar with multiple spiral arms, while the latter contracts but does not collapse. The center of the cloud in model mag-x-1000 does shift along the $\hat{x}$ axis during its lengthy (over $20 t_{f f}$ ) evolution, which is permitted because of the assumption of periodic boundary conditions for these models. While the initial cloud is axisymmetric, non-axisymmetry arises during the evolutions as a result of growth of numerical round-off error, accounting for the asymmetric appearance of many of the models that were evolved for long times. The motion of the center of mass of the cloud for model mag-x-1000 is along the direction of the initial $(\hat{x})$ magnetic field lines, so this overall motion is consistent with movement along field lines. A considerably smaller lateral shift is evident for model mag-z-1800 (Figure 5b), where the initial magnetic field retards such motions.

Flow down magnetic field lines compared to flow across field lines leads to differing effects, as can be seen in Figure 8, which compares the vertical density distributions for two models which both collapsed to form single protostars, models mag-z-1600 (Figure 8a) and mag-x-800 (Figure 8b). Figure $8 \mathrm{a}$ shows that the mag-z-1600 cloud collapsed along the $\hat{z}$ axis, to form a fairly symmetrical distribution about the $z=0$ plane, as both the initial rotation and magnetic fields permit collapse into this plane. Figure $8 \mathrm{~b}$ shows that model mag-x-1600 did collapse primarily into the $z=0$ plane, as permitted by the initial rotation axis, but the vertical distribution is considerably less symmetrical about the $z=0$ plane, as a result of the resistance of the initial magnetic field to collapse along the $\hat{z}$ axis.

Table 2 shows that for initial 50\% density perturbations, the initial magnetic field direction compared to the rotation axis has a profound effect on the outcome of the collapse. We can define several critical initial magnetic field strength values for these initial conditions: $B_{\text {coll }}$ is the maximum field strength permitting sustained collapse, while $B_{\text {frag }}$ is the 
maximum field strength permitting collapse leading to fragmentation. These two critical values differ significantly depending on the initial field orientation: for fields aligned with the rotation axis, $B_{\text {coll }} \sim 1700 \mu \mathrm{G}$ and $B_{\text {frag }} \sim 1550 \mu \mathrm{G}$, whereas for fields initially aligned perpendicular to the rotation axis, $B_{\text {coll }} \sim 900 \mu \mathrm{G}$ and $B_{\text {frag }} \sim 500 \mu \mathrm{G}$. The fact that both critical values are considerably lower in the latter case than in the former can be qualitatively understood in part as being the result of the ability of the dense cloud core to collapse relatively unimpeded to the $z=0$ midplane (unopposed by rotation or magnetic fields) in the former case, compared to the inhibition to collapse to the $z=0$ midplane when the field is initially perpendicular to the rotation axis in the latter case. The extent of angular momentum loss suffered due to magnetic braking in these clouds (Table 2) is clearly also a factor, as for both field orientations, once the total angular momentum loss reaches $\sim 50 \%$, fragmentation into a binary or multiple system is stymied. That this angular momentum loss occurs for smaller initial field strengths than for the perpendicular field alignment implies that such perpendicular fields can be more efficient at magnetic braking, i.e., stretching of the initial $B_{x}$ field lines transports angular momentum outward more efficiently than does twisting of the initial $B_{z}$ field lines.

These results provide a quantitative estimate of how the initial magnetic field value can lead to significantly different outcomes for dense cloud core evolutions. For comparison, observational Zeeman effect estimates of the line-of-sight component of the magnetic field in dense cloud cores with number densities appropriate for these (BB79) models $\left(\sim 10^{7} \mathrm{~cm}^{-3}\right)$ are on the order of $B_{l o s} \sim 1000 \mu \mathrm{G}$ (see Figure 6 in Crutcher 2012), making these critical field strengths of more than theoretical importance for real molecular cloud cores.

It is also interesting to compare these results with the magnetic field strength that separates formally magnetically subcritical clouds from magnetically supercritical clouds (e.g., Nakano \& Nakamura; Crutcher 2012). For a uniform density cloud threaded by a uniform magnetic field, the critical field strength is $B_{c} \approx 2 G^{-1 / 2} M / R^{2}$. For the BB79 cloud, $B_{c} \approx 1000 \mu \mathrm{G}$, which falls between the maximum initial field strengths for collapse (Table 2) of $B_{\text {coll }} \sim 1700 \mu \mathrm{G}$ for fields aligned with the rotation axis, and $B_{\text {coll }} \sim 900 \mu \mathrm{G}$ for perpendicular fields. Note that based on the Price \& Bate (2007) values for the critical mass to flux ratio, this critical field strength would be $\sim 800 \mu \mathrm{G}$, just slightly lower. The numerical models thus straddle the expectations based on the analytic criterion for non-rotating clouds, where the field orientation can have no effect, as well as based on the initial magnetic flux ratio values given in the Tables. 


\subsection{BB79-10 MHD Results}

We now turn to the models listed in Table 3, which are identical to those just discussed, except for having a smaller (10\%) initial density perturbation, as in the BB79-10 models. The same three general types of outcomes are found for these models, as noted above and as listed in Table 3. These models were restricted to having the magnetic field aligned with the rotation axis.

The key result is that the critical magnetic field strength values (Table 3) are significantly different from those obtained with the initial $50 \%$ density perturbations (Table 2). The critical values of $B_{\text {coll }} \sim 1700 \mu \mathrm{G}$ and $B_{\text {frag }} \sim 1550 \mu \mathrm{G}$ for $50 \%$ perturbations decrease to $B_{\text {coll }} \sim 1350 \mu \mathrm{G}$ and $B_{\text {frag }} \sim 1050 \mu \mathrm{G}$ for $10 \%$ perturbations, reflecting the fact that with smaller initial density perturbations, the magnetic fields must be substantially smaller in order to still achieve collapse and fragmentation, though still in the range of observed dense cloud cores (e.g., Crutcher 2012).

\section{Barotropic MHD Results}

Collapsing clouds that are assumed to remain isothermal are considerably more likely to undergo fragmentation than clouds where compressional heating begins to raise the cloud's temperature above $\sim 10 \mathrm{~K}$ at densities above $\sim 10^{-14} \mathrm{~g} \mathrm{~cm}^{-3}$ (e.g., Boss 1986). Accordingly, we now turn to the results for the MHD models with the same barotropic equation employed by Price \& Bate (2007) and Bürzle et al. (2011) in their 3D MHD models of the collapse of uniform density spheres. These models should afford a more realistic assessment of the prospects for magnetic cloud collapse and fragmentation than the isothermal models, at least for clouds which collapse into the nonisothermal regime before fragmenting. Table 4 shows the results for the barotropic MHD models. All of the barotropic models started with $B_{z}<B_{c} \sim 1000 \mu \mathrm{G}$, i.e., initially magnetically supercritical, so all of these models collapsed. The goal of this series was to determine $B_{\text {frag }}$, rather than $B_{\text {coll }}$, and to compare $B_{\text {frag }}$ with the previous results.

Figure 9 shows the evolution of model poly-z-20, which collapsed to form an intermediate central object with spiral arms (Figure 9b), which continue to grow as further mass collapsed to the midplane, leading to fragment formation near the ends of the two-armed spiral (Figure 9c). One of these two new fragments merged with the central object, while the second object was still in orbit at the final time shown (Figure 9d).

Figure 10 shows the evolution of model poly-z-81, which collapsed to form an intermediate central bar (Figure 10b). The bar evolved into a central object surrounded by spiral 
arms (Figure 10c), but in this case, the accretion of further mass into the midplane did not result in the formation of additional clumps: at the same final time as model poly-z-20, a single central object with spiral arms is evident (Figure 10d).

Models poly-z-20 and poly-z-81 represent the two types of outcomes for this set of models (Table 4): collapse in all cases, but sustained fragmentation only for the models with $B_{z}<B_{\text {frag }} \sim 70 \mu \mathrm{G}$. This value for $B_{\text {frag }}$ is considerably lower than those obtained for the previous sets of isothermal MHD models, a clear indication of the added difficulty for fragmentation when the cloud is allowed to heat up upon becoming optically thick at infrared wavelengths at $\rho \sim 10^{-14} \mathrm{~g} \mathrm{~cm}^{-3}$.

This critical value of $B_{\text {frag }} \sim 70 \mu \mathrm{G}$ is also somewhat lower than the critical value found in two previous 3D MHD studies using the barotropic pressure relationship with similar initial conditions. Price \& Bate (2007) found $B_{\text {frag }} \sim 180 \mu \mathrm{G}$ for initial fields aligned with the rotation, as is the case here (Table 4 ), and $B_{\text {frag }} \sim 95 \mu \mathrm{G}$ when the field was perpendicular to the rotation axis. Bürzle et al. (2011) found $B_{\text {frag }} \sim 300 \mu \mathrm{G}$ for initial fields aligned with the rotation. The reason for the differences in $B_{\text {frag }}$ between these two previous studies is unclear, as the later study was intended to duplicate the earlier study, and both studies used SPH MHD codes with the same barotropic relation, starting from identical initial conditions. However, Bürzle et al. (2011) suggested that one reason for the differences could be due to the different MHD techniques employed, resulting in different degrees of "magnetic cushioning", which could aid fragmentation. In the present models, magnetic cushioning does not appear to play as large a role, as the maximum absolute values of $B_{x}$ and $B_{y}$ are about three times as large as $B_{z}$ in the midplanes of both models poly-z-60 and poly-z-81 at their final times, when the former had fragmented, while the latter had not (Figure 10d). These maximum field strengths are all significantly higher (factors of 2 to 3 ) in model poly-z-81 than in polyz-60 at these final times, in spite of their initial field strengths being similar. These final field strengths presumably then are the main reason for the different outcomes in models poly-z-60 and poly-z-81.

Besides the different MHD techniques employed, the differences between these two previous SPH studies and the models shown in Table 4 are likely to be due in part to the relatively modest spatial resolution employed in the present calculations, compared to the use of $\sim 3 \times 10^{6}$ particles in the SPH calculations, combined with the use of sink cells in the SPH models. These sink cells are inserted in both sets of SPH models once the density exceeds $10^{-10} \mathrm{~g} \mathrm{~cm}^{-3}$. While it is not clear from the figures presented in the two SPH papers, the sink cells appear to be inserted in both calculations at about the phase of evolution seen in Figure 10b, for models with initial $B_{z}=81 \mu \mathrm{G}$ for all three codes. The maximum midplane density for model poly-z-81 seen in Figure $10 \mathrm{~b}$ is only $\sim 10^{-13} \mathrm{~g} \mathrm{~cm}^{-3}$, much less than the 
sink cell critical density, so clearly the SPH codes are resolving much higher densities during these collapses than are being resolved with Enzo with the moderate resolution employed. This appears to be the main reason for the lower value of $B_{\text {frag }}$ for the poly-z models, as other differences with the SPH models (e.g., small differences in the initial cloud density, radius, temperature, and rotation rate) appear to be less important. At any rate, the differences in the $B_{\text {frag }}$ values between the poly-z models and the SPH results are comparable to the differences between the two SPH codes themselves; overall, all three codes are in good agreement about the outcome of these 3D MHD collapse and fragmentation models.

\section{Conclusions}

We have tested the Enzo AMR code on a variety of 3D HD and MHD test cases for protostellar collapse, and shown that the Enzo code performs reliably, even with only moderate spatial resolution, and yields results in good agreement with previous studies. We have tested the performance of various hydrodynamical methods on the Boss \& Bodenheimer (1979) standard isothermal test case, and determined the degree to which the various methods conserve mass and angular momentum. The MHD models have led to estimates of the critical initial magnetic field strengths $\left(B_{\text {coll }}\right)$ that separate contraction from collapse (i.e., magnetically subcritical versus supercritical clouds), as well as the strengths $\left(B_{\text {frag }}\right)$ that separate collapse to form a single protostar from those that lead to fragmentation into a binary or multiple protostar system. The different fragmentation outcomes depend in part on the amount of angular momentum lost by magnetic braking during the evolutions, with the isothermal MHD models implying that magnetic braking is more efficient for perpendicular than for aligned fields. The numerically determined values of $B_{\text {coll }} \sim 1700 \mu \mathrm{G}$ for initial fields aligned with the rotation axis and $B_{\text {coll }} \sim 900 \mu \mathrm{G}$ for perpendicular fields compare well with observational estimates of $B_{\text {los }} \sim 1000 \mu \mathrm{G}$ for the densest molecular cloud cores, implying that factors such as the relative orientation of the magnetic field and the rotation axis could have an important effect on cloud collapse.

The initially uniform density, initially uniform rotation (non-turbulent) spheres considered here are convenient for theoretical comparisons, but are not particularly similar to observed molecular cloud cores, which are typically centrally-condensed (e.g., Ward-Thompson, Motte, \& André 1999; Schnee et al. 2010; Butler \& Tan 2012), highly non-spherical, and turbulent. In particular, prolate and oblate shapes have been inferred from observations of suspected pre-collapse molecular cloud cores (e.g., Jones, Basu, \& Dubinski 2001; Curry \& Stahler 2001; Cai \& Taam 2010).

Armed with the experience gained from the present set of MHD models, our future 
Enzo AMR MHD models will consider the collapse of more realistic, centrally-condensed, spheroidal molecular cloud cores, such as the initially prolate and oblate cloud cores considered by Boss (2009), using a pseudo-MHD code to model magnetic field effects and ambipolar diffusion in a variety of clouds (e.g., Boss 2007, 2005, and references therein). We hope to learn if these pseudo-MHD calculations produced results similar to what can be obtained with a true MHD code like Enzo.

We thank Tom Abel, Greg Bryan, Nathan Goldbaum, Craig Lage, Brian O'Shea, Britton Smith, and Matt Turk for advice about the Enzo and yt codes, and the referee, Sven Van Loo, for a large number of great suggestions for improving the manuscript. The computations were performed using the Enzo code developed by the Laboratory for Computational Astrophysics at the University of California San Diego (http://lca.ucsd.edu). The calculations were performed on the Carnegie Xenia Cluster, the purchase of which was partially supported by the National Science Foundation (NSF) under grant MRI-9976645. This work

was partially supported by NSF grant AST-1006305. We also thank Michael Acierno and Ben Pandit for their invaluable assistance with the Xenia cluster.

\section{REFERENCES}

Arreaga-Garcia, G., Klapp, J., Sigalotti, L. D. G., Gabbasov, R. 2007, ApJ, 666, 290

Balsara, D. 2001, J. Comp. Phys. 174, 614

Banerjee, R., \& Pudritz, R. E. 2006, ApJ, 641, 949

Bate, M. R., Bonnell, I. A., \& Price, N. M. 1995, MNRAS, 277, 362

Black, D. C., \& Bodenheimer, P. 1976, ApJ, 206, 138

Boss, A. P. 1986, ApJS, 62, 519

Boss, A. P. 1997, ApJ, 483, 309

Boss, A. P. 2005, ApJ, 622, 393

Boss, A. P. 2007, ApJ, 658, 1136

Boss, A. P. 2009, ApJ, 697, 1940

Boss, A. P., \& Bodenheimer, P. 1979, ApJ, 234, 289

Boss, A. P., Fisher, R. T., Klein, R. I., \& McKee, C. F. 2000, ApJ, 528, 325

Burkert, A., \& Bodenheimer, P. 1993, MNRAS, 264, 798

Bürzle, F., et al. 2011, MNRAS, 412, 171 
Butler, M. J., \& Tan, J. C. 2012, ApJ, 754, 5

Cai, M. J., \& Taam, R. E. 2010, ApJL, 709, L79

Colella, P. \& Woodward, P. R. 1984, J. Comp. Phys., 54, 174

Collins, D. C., Xu, H., Norman, M. L., Li, H., \& Li, S. 2010, ApJS, 186, 308

Crutcher, R. M. 2012, ARAA, 50, 29

Cunningham, A. J., Frank, A., Varniére, P., Mitran, S., \& Jones, T. W. 2009, ApJS, 182, 519

Curry, C. L., \& Stahler, S. W. 2001, ApJ, 555, 160

Dedner,A., Kemm, F., Kröner, D., Munz, C. D., Schnitzer, T., \& Wesenberg, M. 2002, J. Comp. Phys., 175, 645

Duffin, D.F., \& Pudritz, R. E. 2009, ApJL, 706, L46

Federrath, C., Sur, S., Schleicher, D. R. G., Banerjee, R., \& Klessen, R. S. 2011, ApJ, 731, 62

Godunov, S. K. 1959, Matematicheskii Sbornik, 47, 271

Hennebelle, P., \& Fromang, S. 2008, A\&A, 477, 9

Hennebelle, P., \& Teyssier, R. 2008, A\&A, 477, 25

Hennebelle, P., et al. 2011, A\&A, 528, A72

Jones, C. E., Basu, S., \& Dubinski, J. 2001, ApJ, 551, 387

Joos, M., Hennebelle, P., \& Ciardi, A. 2012, A\&A, 543, A128

Kitsionis, S., \& Whitworth, A. P. 2002, MNRAS, 330, 129

Machida, M. N., Tomisaka, K., Matsumoto, T., \& Inutsuka, S.-I 2008, ApJ, 677, 327

Mignone, A., Zanni, C., Tzeferacos, P., van Straalen, B., Colella, P., \& Bodo, G. 2012, ApJS, 198,7

Miniati, F., \& Martin, D. F. 2011, ApJS, 195, 5

Myhill, E. A., \& Boss, A. P. 1993, ApJS, 89, 345

Nakano, T., \& Nakamura, T. 1978, PASJ, 30, 671

Price, D. J., \& Bate M. R. 2007, MNRAS, 377, 77

Ramsey, J. P., Clarke, D. A., \& Men'shchikov, A. B. 2012, ApJS, 199, 13

Schnee, S., et al. 2010, ApJ, 718, 306

Sigalotti, L. D. G. 1997, A\&A, 328, 586 
Stone, J. M., \& Norman, M. L. 1992, ApJS, 80, 753

Teyssier, R. 2002, A\&A, 385, 337

Troland, T. H., \& Crutcher, R. M. 2008, ApJ, 680, 457

Truelove, J. K. Klein, R. I., McKee, C. F., Holliman, J. H., Howell, L. H., \& Greenough, J. A. 1997, ApJ, 489, L179

Turk, M. J., et al. 2011, ApJS, 192, 9 (16pp)

van der Holst, B., et al. 2011, ApJS, 194, 23

Wang, P., \& Abel, T. 2009, ApJ, 696, 96

Wang, P., Abel, T., \& Zhang, W. 2008, ApJS, 176, 467

Ward-Thompson, D., Motte, F., \& André, P. 1999, MNRAS, 305, 143

Ziegler, U. 2008, Comp. Phys. Commun., 179, 227 
Table 1. Percentage change in the total mass $\left(M_{t o t}\right)$ and total angular momentum $\left(J_{t o t}\right)$ during the evolutions of the isothermal HD models, showing the dependence on the hydrodynamic method chosen in several variations of model BB79.

\begin{tabular}{cccc}
\hline \hline model & hydro & $\Delta M_{\text {tot }}$ & $\Delta J_{\text {tot }}$ \\
\hline BB79 & ZEUS & $-0.7 \%$ & $-5 \%$ \\
BB79-HM-0 & PPM & $-0.5 \%$ & $-2 \%$ \\
BB79-HM-4 & MUSCL & $-0.7 \%$ & $-4 \%$ \\
BB79-10 & MUSCL & $-0.5 \%$ & $-2 \%$ \\
BB79-10-HR & MUSCL & $-0.1 \%$ & $-0.2 \%$ \\
\hline
\end{tabular}


Table 2. Initial magnetic field strengths (in microGauss), initial mass to flux ratios $M / \Phi$ (in units of the critical ratio), and the results (at the final time $t_{f}$, in free fall units) for the isothermal MHD models with initial $50 \%$ density perturbations, as in the non-magnetic model BB79.

\begin{tabular}{ccccccc}
\hline \hline model & $B_{x}(\mu \mathrm{G})$ & $B_{z}(\mu \mathrm{G})$ & $M / \Phi$ & $t_{f} / t_{f f}$ & $\Delta J_{\text {tot }}$ & result \\
\hline mag-z-300 & 0 & 300 & 2.7 & 1.8 & $-5.0 \%$ & collapses/fragments \\
mag-z-600 & 0 & 600 & 1.3 & 2.4 & $+3.1 \%$ & collapses/fragments \\
mag-Z-1000 & 0 & 1000 & 0.80 & 1.7 & $-5.9 \%$ & collapses/fragments \\
mag-z-1200 & 0 & 1200 & 0.67 & 2.4 & $-8.6 \%$ & collapses/fragments \\
mag-z-1300 & 0 & 1300 & 0.62 & 2.2 & $-9.1 \%$ & collapses/fragments \\
mag-Z-1400 & 0 & 1400 & 0.57 & 2.6 & $-13 . \%$ & collapses/fragments \\
mag-Z-1500 & 0 & 1500 & 0.53 & 6.0 & $-39 . \%$ & collapses/fragments \\
mag-z-1600 & 0 & 1600 & 0.50 & 7.7 & $-49 . \%$ & collapses/single \\
mag-Z-1800 & 0 & 1800 & 0.44 & 9.2 & $-57 . \%$ & little collapse/single \\
mag-Z-2000 & 0 & 2000 & 0.40 & 11.5 & $-63 . \%$ & little collapse/single \\
mag-X-300 & 300 & 0 & 2.7 & 5.8 & $-0.90 \%$ & collapses/fragments \\
mag-X-400 & 400 & 0 & 2.0 & 3.2 & $-19 . \%$ & collapses/fragments \\
mag-x-600 & 600 & 0 & 1.3 & 13.4 & $-69 . \%$ & collapses/single \\
mag-X-700 & 700 & 0 & 1.1 & 15.6 & $-77 . \%$ & collapses/single \\
mag-X-800 & 800 & 0 & 1.0 & 8.8 & $-67 . \%$ & collapses/single \\
mag-X-1000 & 1000 & 0 & 0.80 & 20.7 & $-83 . \%$ & little collapse/single \\
mag-X-1200 & 1200 & 0 & 0.67 & 44.0 & $-82 . \%$ & little collapse/single \\
\hline
\end{tabular}


Table 3. Initial magnetic field strengths $B_{z}$ (in microGauss), initial mass to flux ratios $M / \Phi$ (in units of the critical ratio), and the results (at the final time $t_{f}$, in free fall units) for the isothermal MHD models with initial 10\% density perturbations, as in the non-magnetic model BB79-10.

\begin{tabular}{cccccc}
\hline \hline model & $B_{z}(\mu \mathrm{G})$ & $M / \Phi$ & $t_{f} / t_{f f}$ & $\Delta J_{t o t}$ & result \\
\hline mag-Z-400-10 & 400 & 2.0 & 2.2 & $+9.7 \%$ & collapses/fragments \\
mag-z-800-10 & 800 & 1.0 & 2.0 & $-1.2 \%$ & collapses/fragments \\
mag-Z-900-10 & 900 & 0.89 & 3.5 & $-11 . \%$ & collapses/fragments \\
mag-Z-1000-10 & 1000 & 0.80 & 7.3 & $-30 . \%$ & collapses/fragments \\
mag-z-1100-10 & 1100 & 0.73 & 9.5 & $-38 . \%$ & collapses/single \\
mag-z-1200-10 & 1200 & 0.67 & 3.5 & $-19 . \%$ & collapses/single \\
mag-Z-1300-10 & 1300 & 0.62 & 14.0 & $-64 . \%$ & collapses/single \\
mag-z-1400-10 & 1400 & 0.57 & 6.1 & $-40 . \%$ & little collapse/single \\
mag-z-1600-10 & 1600 & 0.50 & 12.8 & $-61 . \%$ & little collapse/single \\
mag-Z-1800-10 & 1800 & 0.44 & 15.8 & $-66 . \%$ & little collapse/single \\
\hline
\end{tabular}


Table 4. Initial magnetic field strength $B_{z}$ (in microGauss), initial mass to flux ratios $M / \Phi$ (in units of the critical ratio), and the results (at the final time $t_{f}$, in free fall units) for the barotropic MHD models, models that are otherwise identical to the non-magnetic, isothermal model BB79-10.

\begin{tabular}{cccccc}
\hline \hline model & $B_{z}(\mu \mathrm{G})$ & $M / \Phi$ & $t_{f} / t_{f f}$ & $\Delta J_{t o t}$ & result \\
\hline poly-z-407 & 407.0 & 2.0 & 1.85 & $-1.8 \%$ & collapses/single \\
poly-z-203 & 203.0 & 4.0 & 2.09 & $-1.1 \%$ & collapses/single \\
poly-z-163 & 163.0 & 5.0 & 1.97 & $-1.9 \%$ & collapses/single \\
poly-z-109 & 109.0 & 7.5 & 2.20 & $-3.8 \%$ & collapses/single \\
poly-z-81 & 81.3 & 10. & 2.20 & $-4.9 \%$ & collapses/single \\
poly-z-60 & 60.0 & 15. & 2.20 & $-4.1 \%$ & collapses/fragments \\
poly-z-41 & 41.0 & 20. & 2.20 & $-3.1 \%$ & collapses/fragments \\
poly-z-20 & 20.0 & 41. & 2.20 & $-3.5 \%$ & collapses/fragments \\
poly-z-10 & 10.0 & 82. & 2.20 & $-2.4 \%$ & collapses/fragments \\
poly-z-5 & 5.0 & 164. & 2.20 & $-3.2 \%$ & collapses/fragments \\
poly-z-0 & 0.0 & $\infty$ & 2.20 & $-1.7 \%$ & collapses/fragments \\
\hline
\end{tabular}



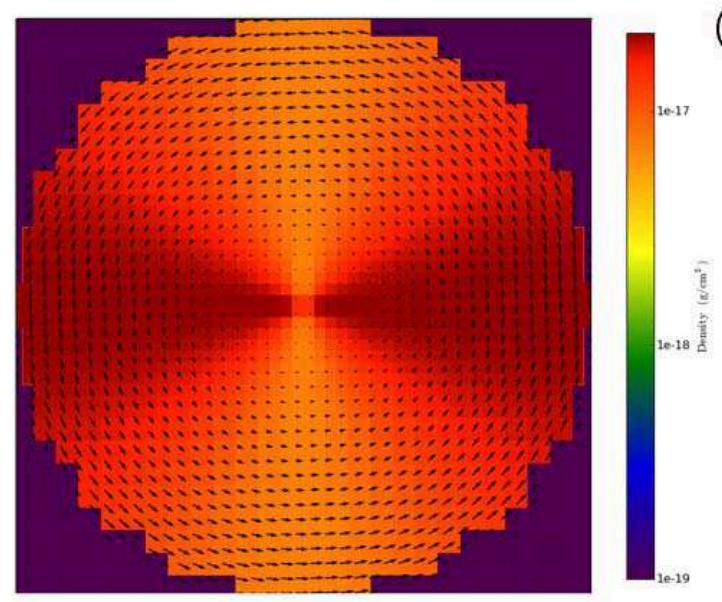

(a)

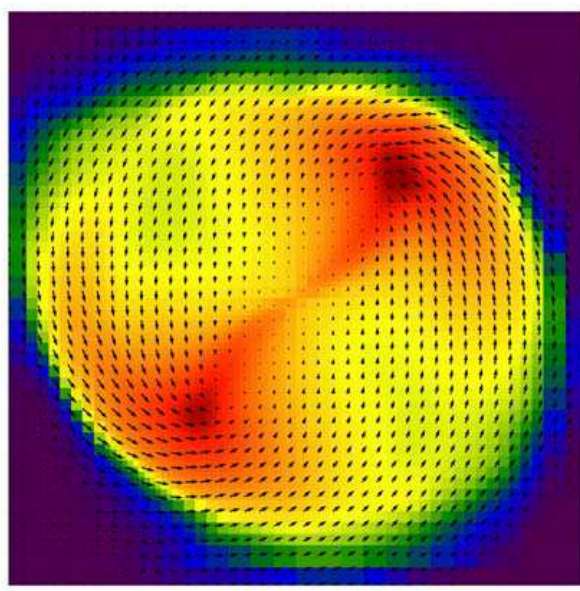

(b)
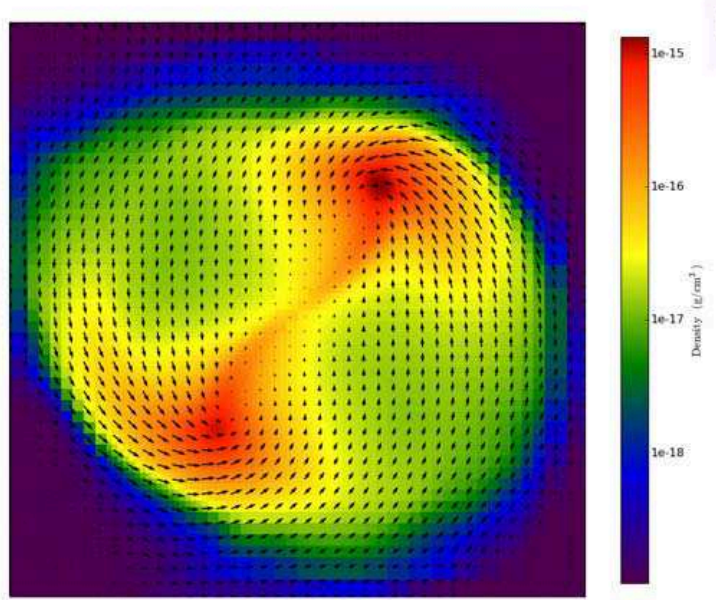

(c)

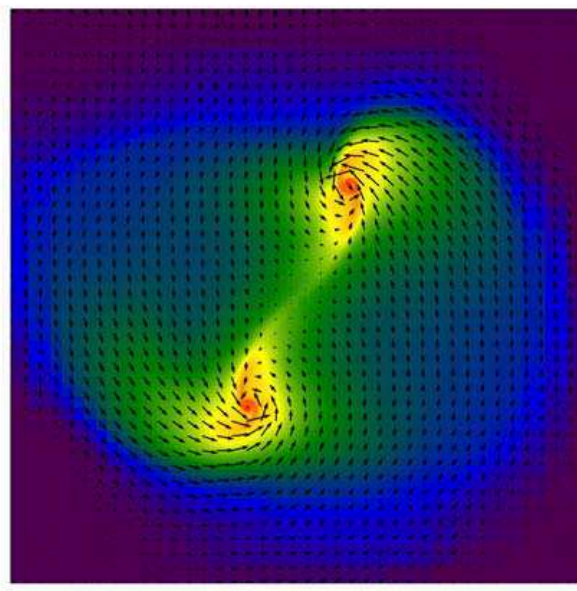

(d)

Fig. 1. - Evolution of the midplane $(z=0)$ density distribution and velocity vectors for the isothermal, non-magnetic model BB79, shown at times: a - 0.0, b - $0.871 t_{f f}, \mathrm{c}-0.950 t_{f f}$, and $\mathrm{d}-1.100 t_{f f}$. Maximum velocities are $\sim 0.5 \mathrm{~km} \mathrm{~s}^{-1}$. Region shown is $6.4 \times 10^{16} \mathrm{~cm}$ across in each case. 

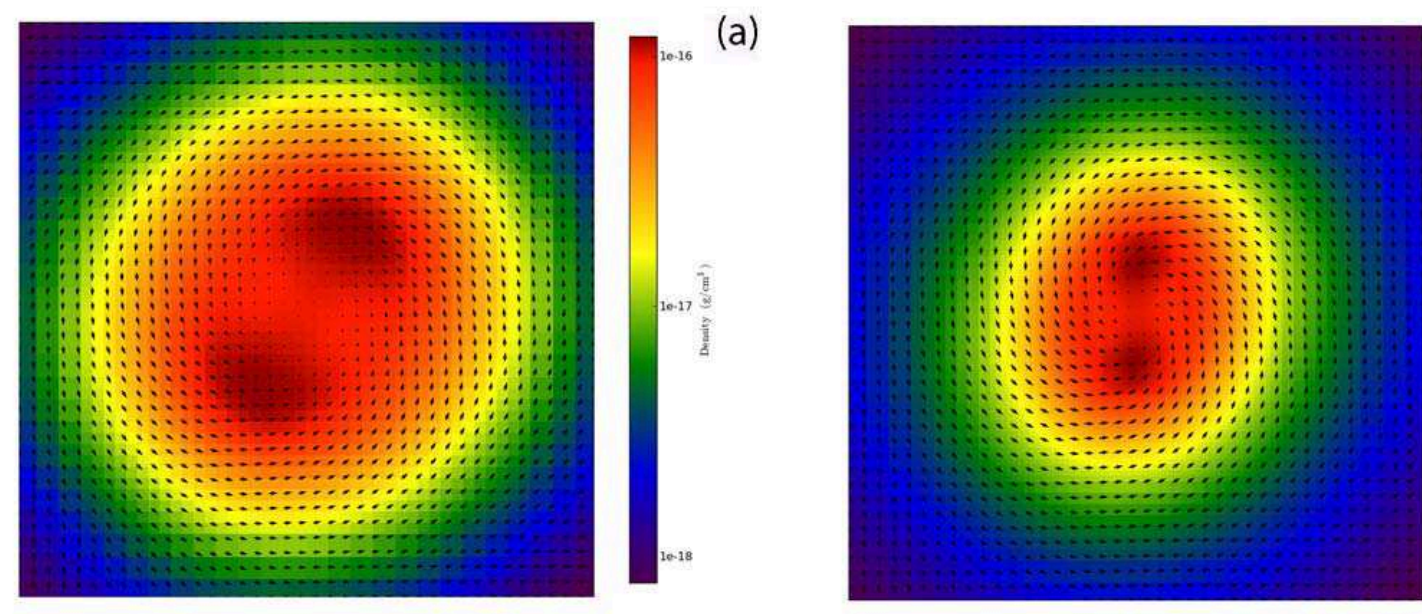

(b)
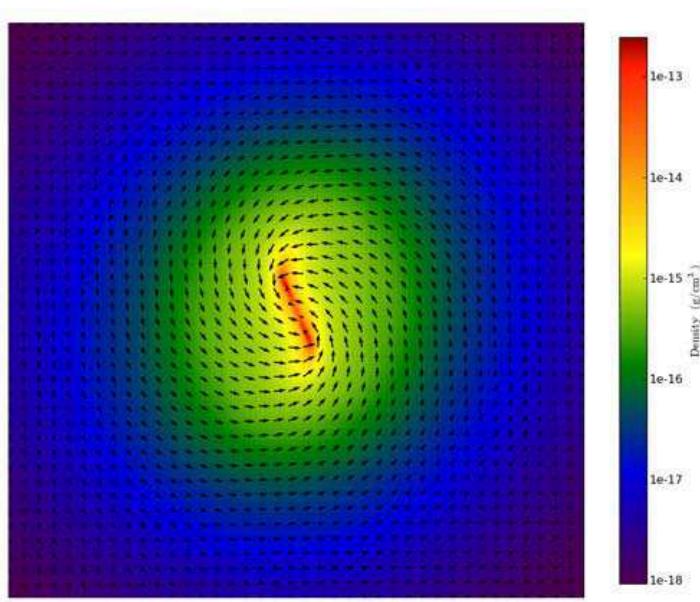

(c)

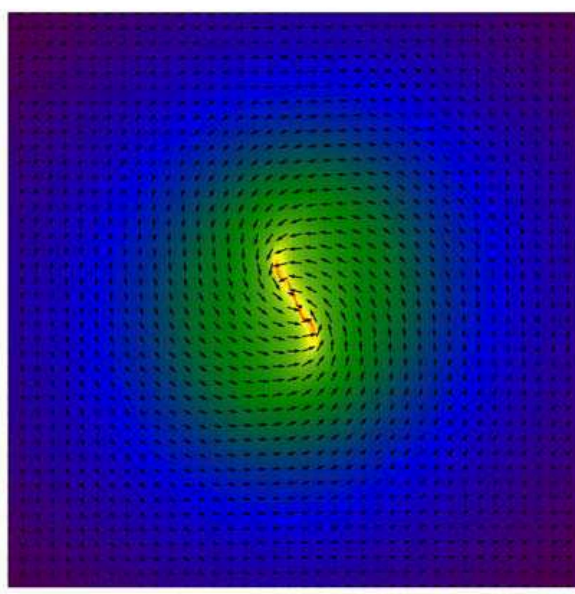

(d)

Fig. 2.- Evolution of the midplane density distribution and velocity vectors for the isothermal, non-magnetic model BB79-10, shown at times: a - $0.990 t_{f f}, \mathrm{~b}-1.230 t_{f f}, \mathrm{c}-1.364 t_{f f}$, and $\mathrm{d}-1.386 t_{f f}$, plotted as in Figure 1. 

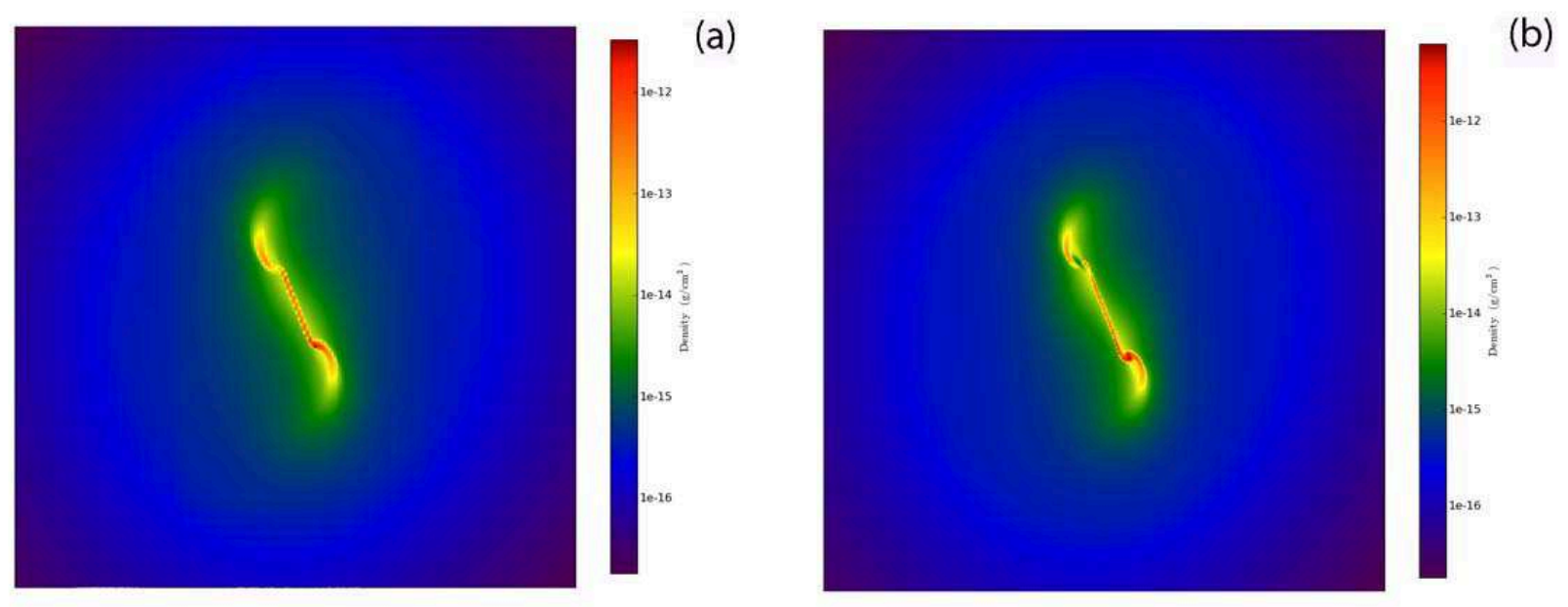

Fig. 3.- Midplane density distributions for model BB79-10 (a) and for model BB79-10-HR (b), both at a time of $1.386 t_{f f}$. Region shown is $3.2 \times 10^{16} \mathrm{~cm}$ across in both cases. 

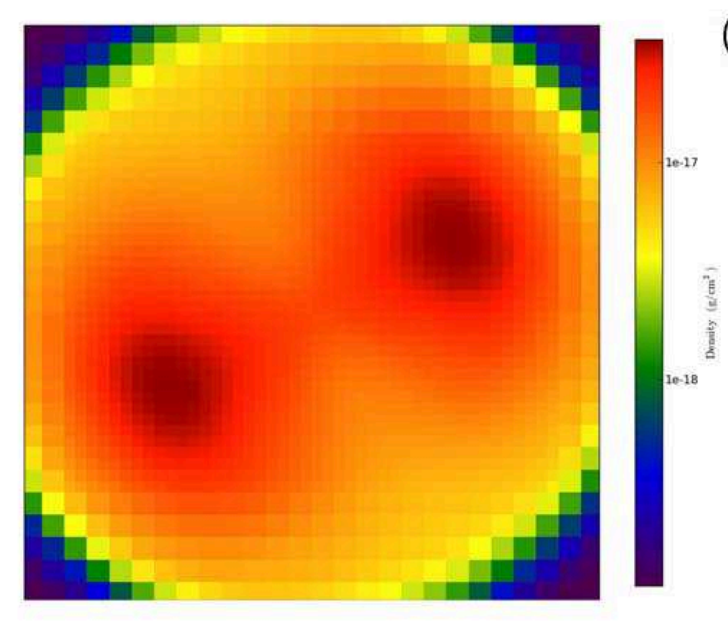

(a)
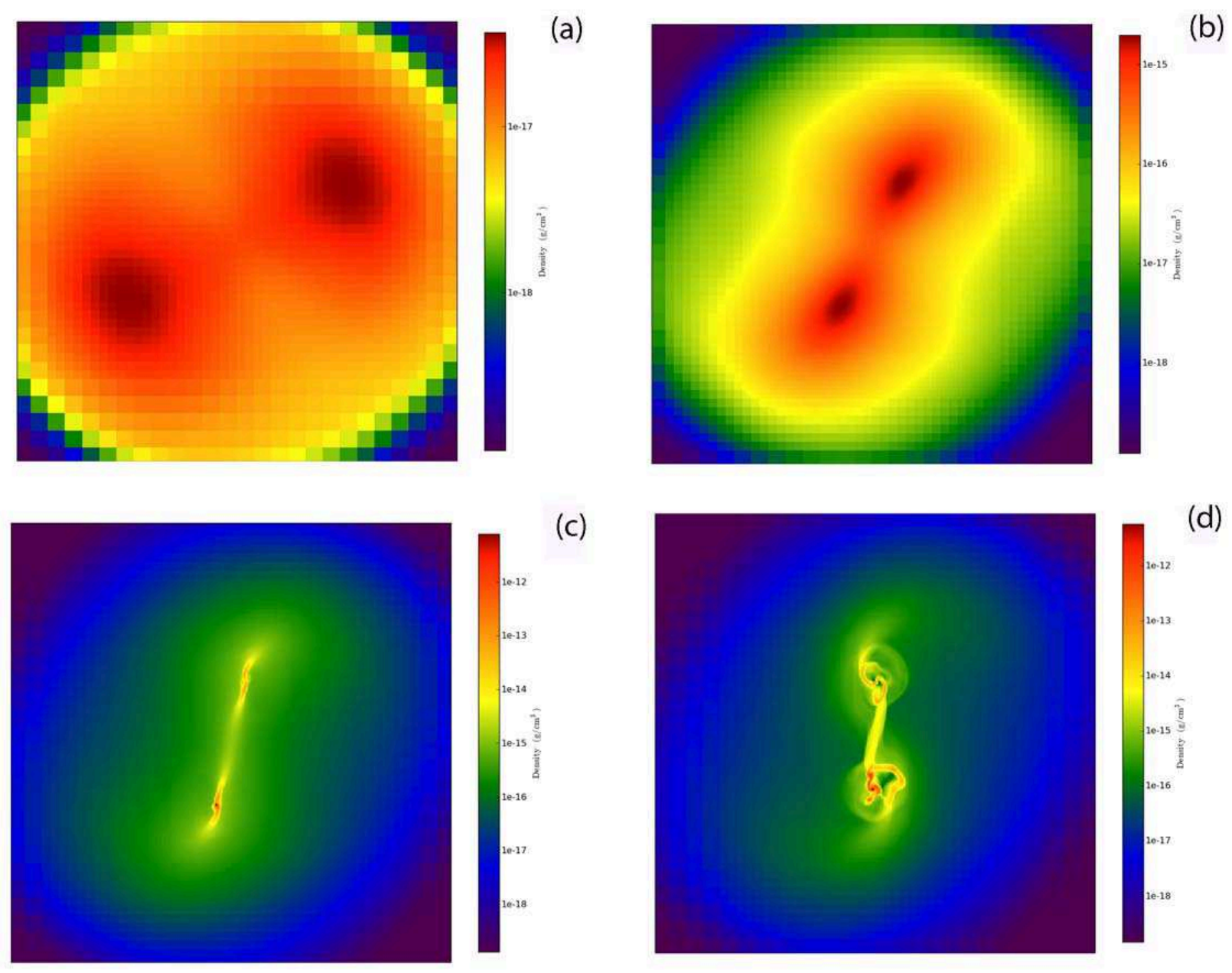

Fig. 4.- Evolution of the midplane density distribution for the isothermal, magnetic model mag-z-300 (a magnetic version of model BB79), shown at times: a $-0.530 t_{f f}, \mathrm{~b}-1.109 t_{f f}$, $\mathrm{c}-1.324 t_{f f}$, and d $-1.577 t_{f f}$, plotted as in Figure 1. 

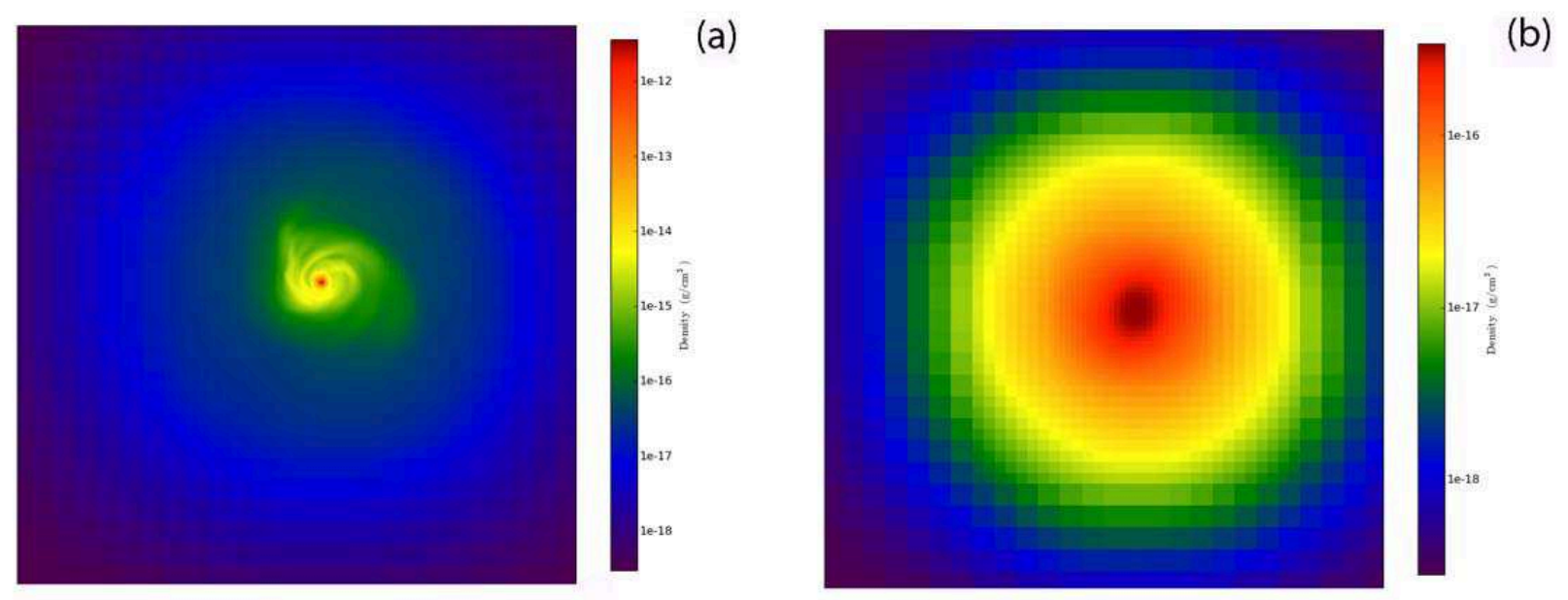

Fig. 5.- Midplane density distributions for models mag-z-1600 (a) and mag-z-1800 (b), at times of 7.742 and $9.350 t_{f f}$, respectively, plotted as in Figure 1. 

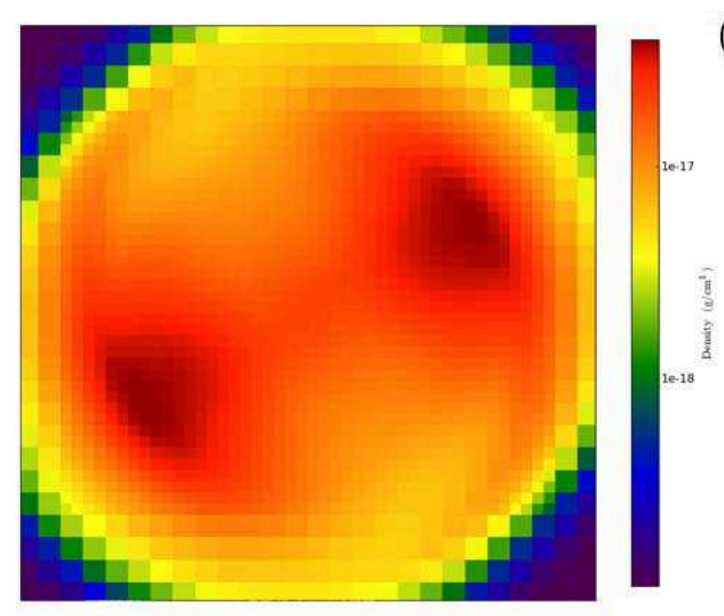

(a)
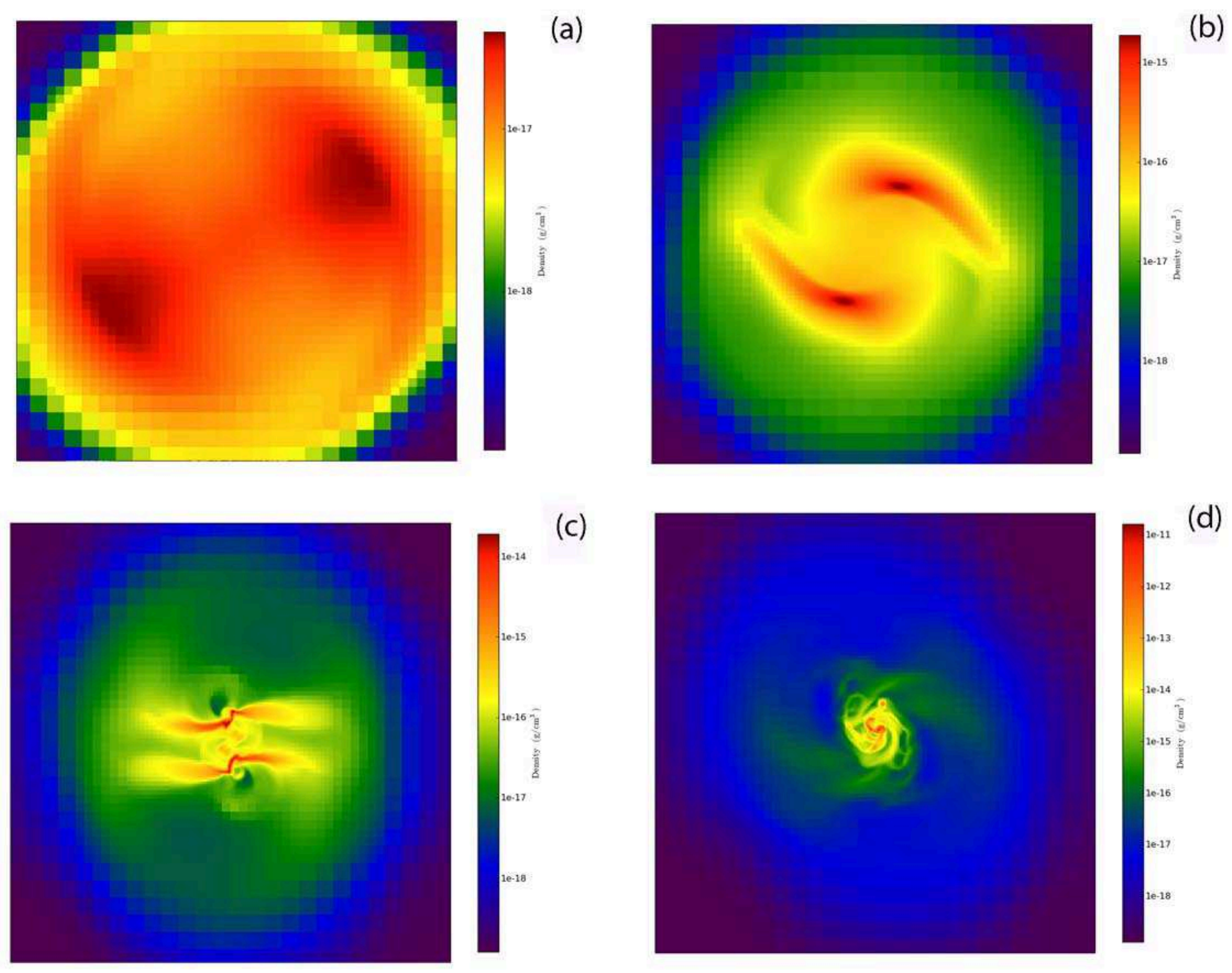

(c)

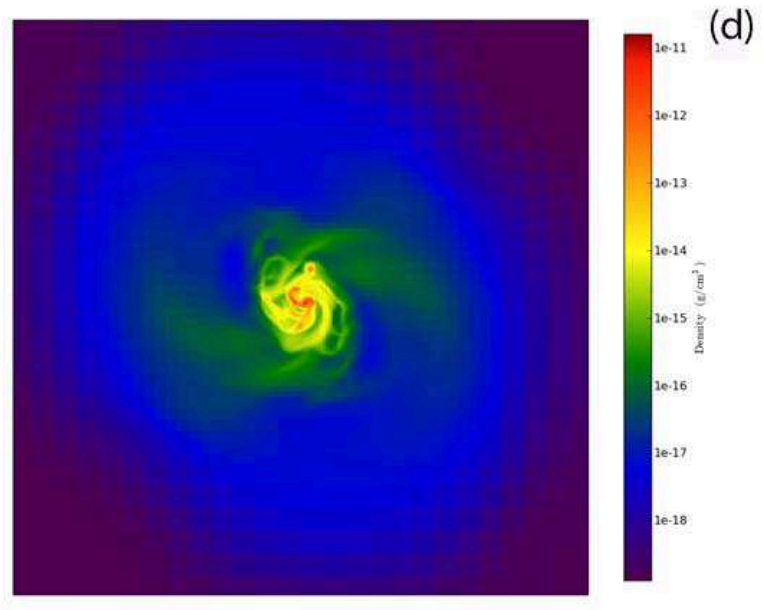

Fig. 6.- Evolution of the midplane density distribution for the isothermal, magnetic model mag-x-300 (a magnetic version of model BB79), shown at times: a $-0.524 t_{f f}, \mathrm{~b}-1.243 t_{f f}$, $\mathrm{c}-1.540 t_{f f}$, and $\mathrm{d}-1.791 t_{f f}$, plotted as in Figure 1. 

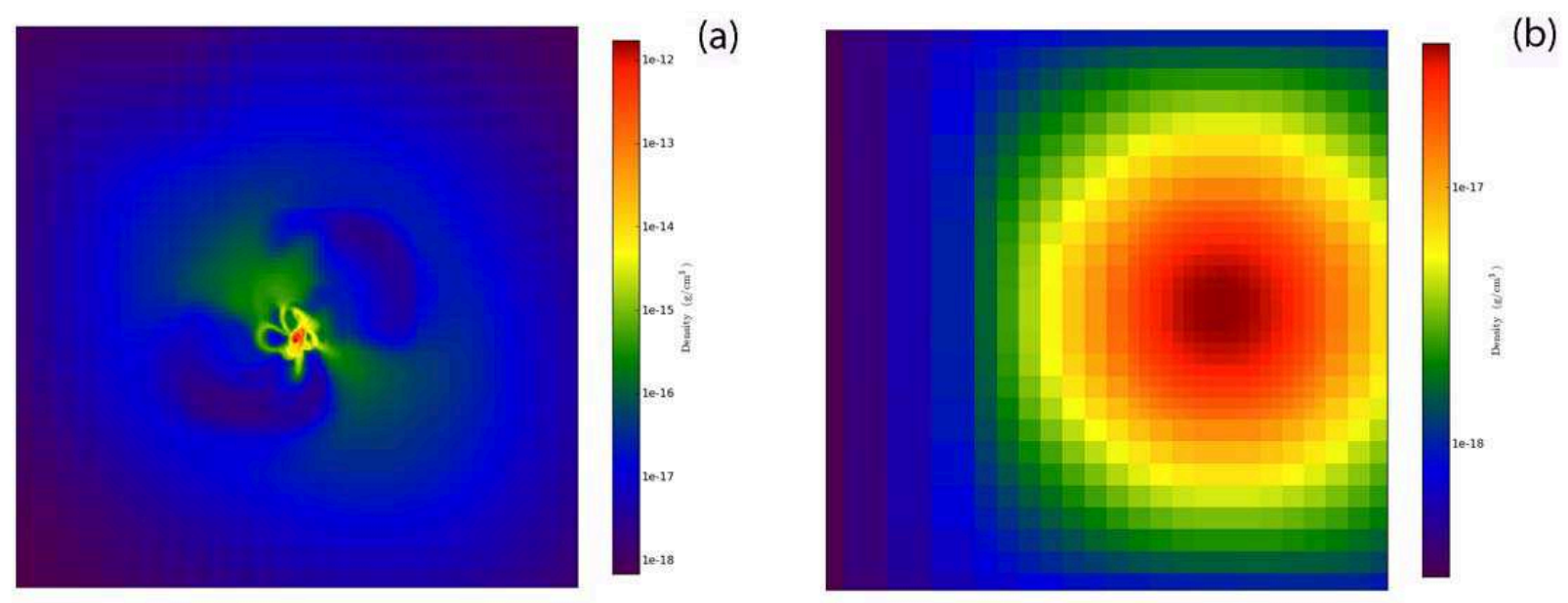

Fig. 7.- Midplane density distributions for models mag-x-800 (a) and mag-x-1000 (b), at times of 8.769 and $20.73 t_{f f}$, respectively, plotted as in Figure 1. 

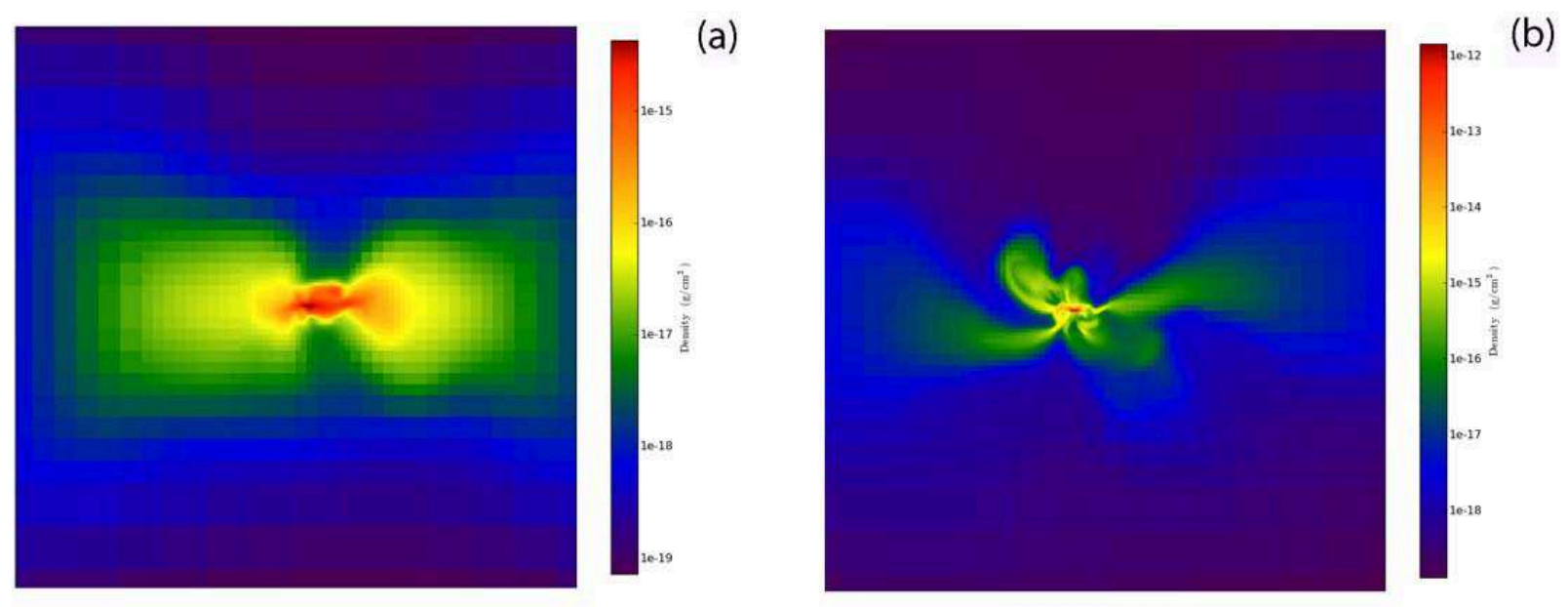

Fig. 8. - Vertical ( $x=0$ plane) density distributions for models mag-z-1600 (a) and mag-x800 (b), at times of 7.742 and $8.769 t_{f f}$, respectively, plotted as in Figure 1. 

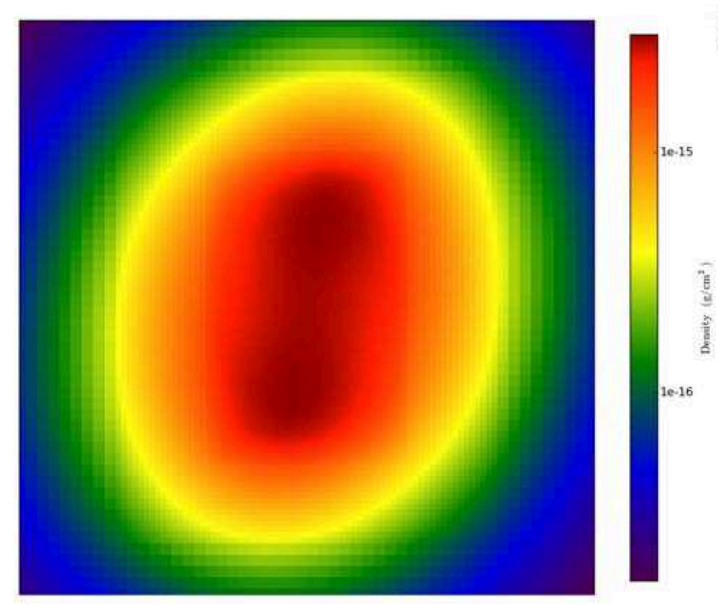

(a)

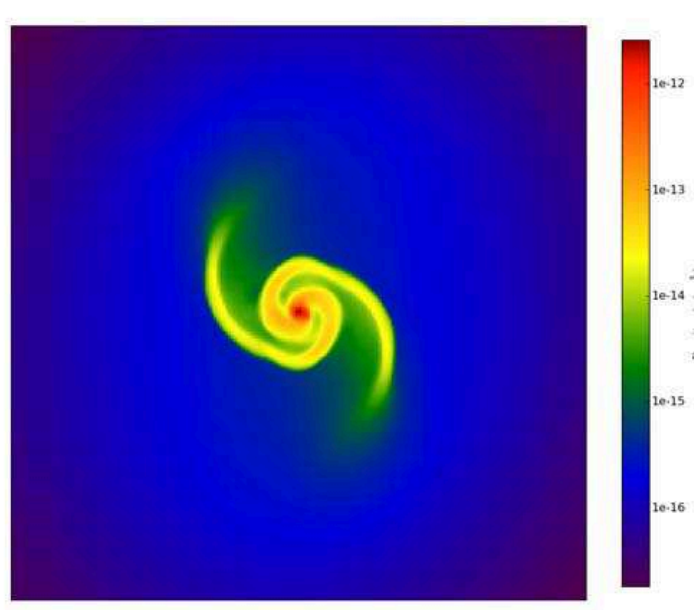

(b)
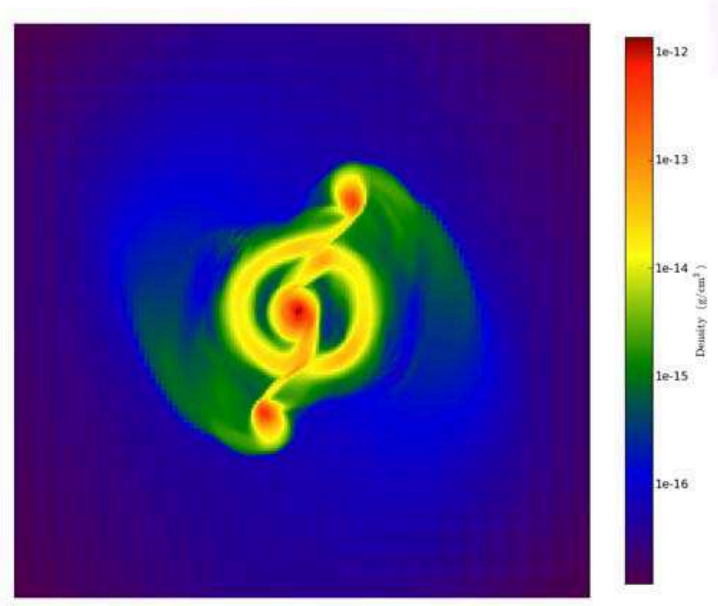

(c)

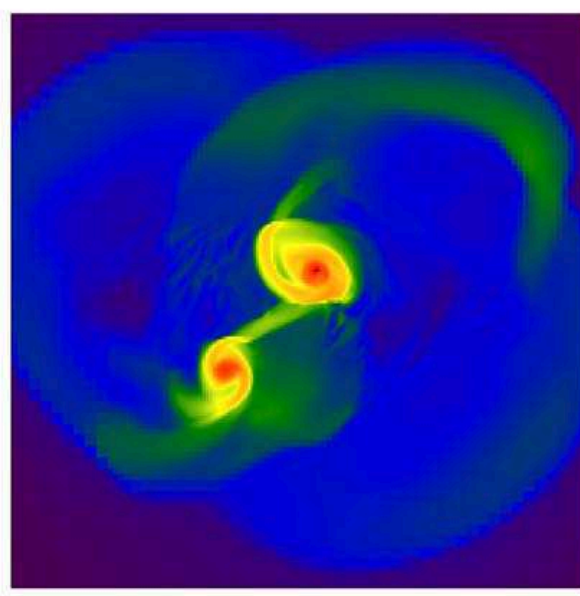

(d)

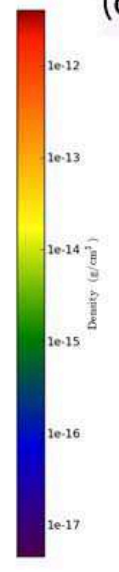

Fig. 9.- Evolution of the midplane density distribution for the barotropic, magnetic model poly-z-20 (a magnetic version of model BB79-10), shown at times: a - $1.179 t_{f f}, \mathrm{~b}-1.503$ $t_{f f}, \mathrm{c}-1.797 t_{f f}$, and $\mathrm{d}-2.200 t_{f f}$. Region shown is $3.2 \times 10^{16} \mathrm{~cm}$ across at all times. 

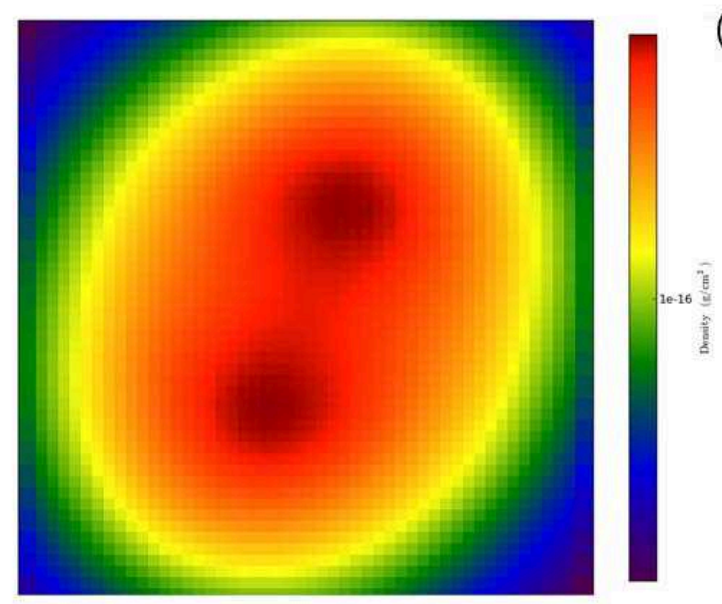

(a)
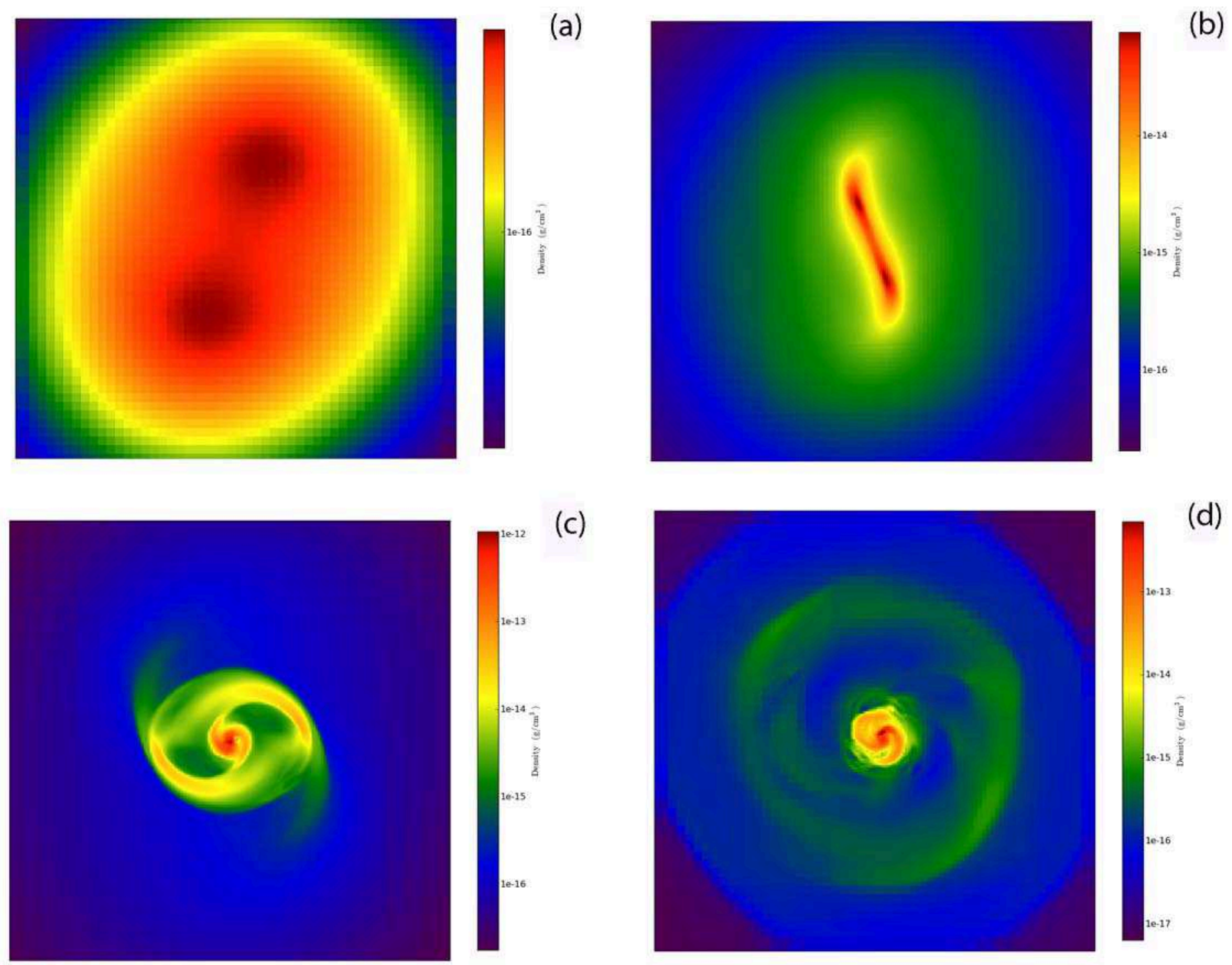

(c)

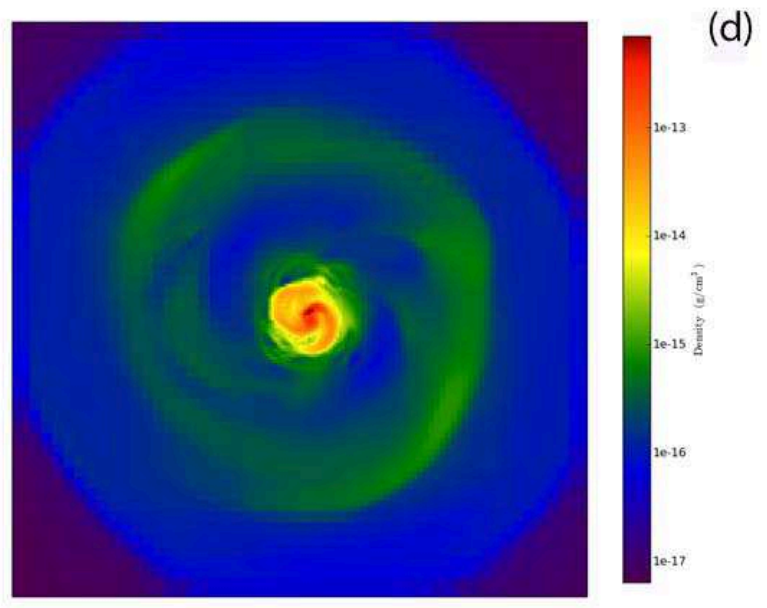

Fig. 10.- Evolution of the midplane density distribution for the barotropic, magnetic model poly-z-81 (a magnetic version of model BB79-10), shown at times: a - $1.089 t_{f f}, \mathrm{~b}-1.362$ $t_{f f}, \mathrm{c}-1.628 t_{f f}$, and $\mathrm{d}-2.200 t_{f f}$. Region shown is $3.2 \times 10^{16} \mathrm{~cm}$ across at all times. 